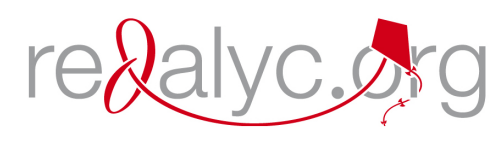

Red de Revistas Científicas de América Latina, el Caribe, España y Portugal

Sistema de Información Científica

Costa, Joana; Marôco, João; Pinto-Gouveia, José; Galhardo, Ana

Validation of the Psychometric Properties of Acceptance and Action Questionnaire-II in Clinical and Nonclinical

Groups of Portuguese Population

International Journal of Psychology and Psychological Therapy, vol. 14, núm. 3, septiembre-diciembre, 2014, pp. 353-364

Universidad de Almería

Almería, España

Available in: http://www.redalyc.org/articulo.oa?id=56032544003

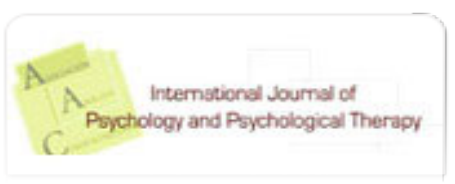

International Journal of Psychology and

Psychological Therapy,

ISSN (Printed Version): 1577-7057

riptp@ual.es

Universidad de Almería

España

How to cite $\mid$ Complete issue $\mid$ More information about this article $\mid$ Journal's homepage

\title{
www.redalyc.org
}

Non-Profit Academic Project, developed under the Open Acces Initiative 


\title{
Validation of the Psychometric Properties of Acceptance and Action Questionnaire-II in Clinical and Nonclinical Groups of Portuguese Population
}

\author{
Joana Costa* \\ Universidade de Coimbra, Portugal \\ João Marôco \\ Unidade de Investigação em Psicologia e Saúde (UIPES), ISPA-IU, Lisboa, Portugal \\ José Pinto-Gouveia \\ Universidade de Coimbra, Portugal \\ Ana Galhardo \\ Instituto Superior Miguel Torga, Coimbra, Portugal
}

\begin{abstract}
This study explores the factor structure of the Acceptance and Action Questionnaire (AAQ-II) in both clinical and general normative groups. It also examines the factorial invariance of a one-factor proposed model in both groups. Data was collected from the overall database of a Portuguese Cognitive and Behavioral Research Center $(N=687,425$ females; mean age $=36$ years; $S D=11.33$ ). Confirmatory Factor Analysis supported a one-factor structure with good internal consistencies and construct related validity. The one-factor solution was also supported with a second independent data set, which showed a configural, strict measurement and structural invariance of the one-factor solution proposed. Multigroup Confirmatory Factorial Analysis showed the configural invariance, weak measurement invariance and also structural invariance of the one-factor model of Acceptance and Action Questionnaire II across both groups under study. The one-factor model have both similar meanings and the same structure, but the measurement model in clinical and nonclinical groups was not the same. Toxic influences of experiencial avoidance as a core mechanism in the development and maintenance of several clinical disorders, may explain why the AAQ-II does not operate equivalently across clinical and nonclinical groups.

Key words: experiential avoidance, one-factor model, factorial invariance, clinical and nonclinical groups.
\end{abstract}

What is already known about the topic?

\section{Novelty and Significance}

Experiential avoidance is critical to the development and maintenance of psychopathology. Psychological problems are not the result of thoughts or feelings themselves but the results of the attempts to suppress, avoid and control such unwanted private events.

- Efforts to avoid unwanted thoughts, feelings and sensations may increase the frequency of these private events and the severity of psychological symptoms.

What this paper adds?

- This study supports the one-factor structure of AAQ-II with strong evidence of psychometric validity.

- This measure shows adequate psychometric properties but also measures the same psychological construct in pathological and normative groups.

- Experiential avoidance has the same meaning for both groups but the factor loadings across them were not equal, with significantly higher factor loadings across clinical group in comparison with nonclinical group.

\footnotetext{
"Correspondence concerning this article: Joana Costa, CINEICC, Faculdade de Psicologia e Ciências da Educação, Universidade de Coimbra, Rua do Colégio Novo, Apartado 6153, 3001-802 Coimbra, Portugal. E-mail: joanascosta@ hotmail.com
} 
A growing body of research has shown important evidences that mental health and behavioral effectiveness are more influenced by how people relate to their internal experiences (e.g. the function of their thoughts, sensations, feelings) than by their form (e.g. how negative their are). In fact, recent developments within cognitive-behaviour therapies promote a shift away from methods that emphasize the control and/or change of the content of psychological experience towards more contextual methods, that emphasize the function of the experience. These new generation of therapies focus precisely on the difference between form and function of private experiences. This is to say that rather than targeting and attempting to alter the content, frequency and/or form of private events, acceptance-based therapies like Acceptance and Commitment Therapy (ACT) seek to alter the context and the function of the internal phenomena (Hayes \& Duckworth, 2006). ACT is based on the assumption that most problems that patients face are due to experiential avoidance (Hayes, Strosahl, Wilson, Bissett, Pistorello, Toormino, et al., 2004). This construct has been operationalized as an individual's unwillingness to experience feelings, physical sensations and thoughts, as well as attempts to alter the form or frequency of these events and the contexts that occasion them (Hayes, Wilson, Gifford, Follette, \& Strosahl, 1996). It is a process involving excessive negative evaluations of unwanted private experiences, an unwillingness to experience these private events and implicates deliberate efforts to control or escape from them (Hayes, 1994; Hayes, Strosahl, \& Wilson, 1999; Kashdan, Barrios, Forsyth, \& Steger, 2006).

Given this definition, experiential avoidance is thought to be critical to the development and maintence of psychopathology (Hayes et al., 1999). More specifically, it has been hypoyhesized that psychological problems are not the result of thoughts or feelings themselves but rather these problems are the results of the attempts to suppress, avoid and control such unwanted private events.

Indeed research has suggested that efforts to avoid unwanted thoughts, feelings and sensations may increase the frequency of these private events and the severity of psychological symptoms. Despite the ultimate maladapative outcome, individuals continue to engage in experiential avoidance because the immediate effects are seemingly positive in that the avoidance strategy initially results in apparent decreases of emotional intensity experiences (Sloan, 2004). However, the pattern of a short term reduction leads to a a self amplifying loop that appears to be resistant to change (Hayes et al., 1999). In fact, experiential avoidance becomes a disordered process when it is appled rigidly and inflexible such that an enourmous time, effort and energy is devoted to managing, controlling or struggling with unwanted private events. According to ACT (Hayes, Strosahl, \& Wilson, 1999) this struggle gets in the way of movement toward valued goals, diminishes contact with present experiences and yields impairment in functioning. The unwillingness to remain in contact with negatively evaluated private events and chronic attempts to alter the form, frequency or content of these events are proposed to be the stronger contributor to psychopatology (Forsyth, Eifert, \& Barrios 2006, Hayes et al., 1999).

The Acceptance and Action Questionnaire (AAQ; Hayes et al., 2004) is the most widely measure of experiential avoidance and psychological inflexibility used. It was originally developed within ACT but now it is also applied to other forms of contextual 
CBT's (Rusch et al., 2008). The primary need for a AAQ-II was that AAQ-I showed insufficient levels of reliability in various populations. The initial item pool in the study of AAQ-II (Bond et al., 2011) was generated by ACT therapists and resaerchers to represent the kind of phenomena that constitutes this unidimensional construct. The final scale contained items on negative evaluations of feelings, avoidance of thoughts and feelings, distinguishing a thought from its referent and behavioral adjustment in the presence of difficult internal experiences such as thoughts, sensations and feelings. An exploratory factor analysis suggested a two-factor solution for a 10-item scale. However the second factor consisted of only the three positively worded items on the scale, suggesting that the second factor resulted from a method (wording) effect and did not represent a substantive dimension. Bond et al., (2011) performed several tests comparing both internal and external validities of the 9 and 16-item scale. Thus, the twofactor soluction was rejected and the three items were not retained in the final structure. Therefore, Bond et al., (2011) concluded that the AAQ-II was an unidimentional measure that assesses the construct of psychological inflexibility. Results indicate that it does so in a comparable manner across different samples (Bond et al., 2011). It is important to notice that Bond et al.'s (2011) research involved six samples with a total of 2816 individuals, 290 of those seek outpatient psychological treatment for substance misuse.

Given all that has been said, this study was designed to address the following aims: 1) to replicate the one-factor structure identified by Bond et al., (2011) in both clinical and general, normative groups; 2) to examine the measurement invariance of the Acceptance and Action Questionnaire-II across these clinical and non clinical group.

\section{Method}

\section{Participants}

Participantes were selected from the databases of a Portuguese research center which include patients who had computed the AAQ-II and that belonged to three major groups of diagnosis (e.g. arthritis rheumatoid, infertility and general population). Analysis of item response patterns did not find any study participants with missing responses to more than half of the experiential avoidance items. For participants with less than $5 \%$ of missing data, missing values were inputed with the regression method. The main sample included 700 participants randomly divide into two groups, a test group formed by 407 individuals (about $60 \%$ of the main sample), and a validation group is composed by 293 individuals (about $40 \%$ of the main sample).

Demographic information of both groups are presented in Table 1. The clinical and general population groups did not show statistically significant differences regarding age and years of school attendance.

\section{Measures}

Acceptance and Action Questionnaire-II (AAQ-II; Bond et al., 2011; Portuguese version: Pinto-Gouveia, Gregório, Dinis, \& Xavier, 2012) is a 7-item self-report 
Table 1. Samples demographic characteristics (Total Sample, $N=697$ ).

\begin{tabular}{|c|c|c|c|c|c|}
\hline & & & $\begin{array}{c}\text { Males } \\
(n=272)\end{array}$ & $\begin{array}{l}\text { Females } \\
(n=425)\end{array}$ & $\chi^{2}$ \\
\hline \multirow{4}{*}{ Marital State } & Single & & 131 & 234 & \multirow{4}{*}{$16.744^{* * * *}$} \\
\hline & Married/ Union & & 141 & 174 & \\
\hline & Divorced & & 0 & 4 & \\
\hline & Widower & & 0 & 13 & \\
\hline \multirow{4}{*}{ Socio-Economic Status } & Low & & 58 & 137 & \multirow{3}{*}{$10.480^{* *}$} \\
\hline & Mean & & 95 & 125 & \\
\hline & High & & 118 & 156 & \\
\hline & $M$ & $S D$ & $\bar{M}$ & $S D$ & $t$ \\
\hline Age & 38.47 & 9.220 & 40.38 & 12.449 & $-2.321^{\prime \prime}$ \\
\hline Education & 12.89 & 4.262 & 12.90 & 5.051 & -.044 \\
\hline
\end{tabular}

questionnaire that assesses experiential avoidance, as efforts to not come into contact with unpleasant private events such as thoughts, feelings, enotions, sensations, by trying to change their occurence, form or frequency specially when doing so leads to undesirable outcomes. Items are rated on a 1 (never true) to 7 (always true) scale; higher results mean high experiential avoidance. AAQ-II has a mean alpha coefficient of .84 (from .78 to .88 ) and the 3 and 12 months teste-retest reliability was .81 and .79 , respectively. The AAQ-II scores concurrently, longitudinally and incrementally predict several outcomes, from mental health to work absence rates. The AAQ-II also shows appropriate discriminat validity and appears to measure the same concept of AAQ-I with better psychometric properties (Bond et al., 2011). In the present study the internal consistency, Cronbach's alpha was .92 .

\section{Procedure}

Relevant database files were initially identified by searching the overall research center databases. Limits were implemented to refine the scope and to ensure quality: databases were limited to adult samples; studies were eligible for inclusion if they included the Acceptance and Action Questionnaire-II (AAQ-II; Hayes et al., 2004) and the patients diagnosis were made by specialists. These data bases were aggregated in clinical and non-clinical group.

\section{Psychometric data evaluation}

To examine whether respondents within the test and validation samples differed with respect to demographic variables, we computed independent Student's $t$-test to compare the two samples on age and education. Pearson's $\chi^{2}$ test was used to compare the two samples on sex, marital status and profession. Kolmogorov-Smirnov tests were used to inspect the data distribution also with a close inspection of the Skewness and Kurtosis values. The presence of multivariate outliers was assessed with the squared Mahanalobis Distance $\left(D M^{2}\right)$

A Confirmatory Factorial Analysis (CFAs) was conducted to confirm the underlying structure of AAQ-II in clinical and and general population groups, using SPSS-AMOS (v.18). Given the good fit of the one-factor model, a Multigroup Confirmatory Factorial 
Analysis was performed to evaluate the level of measurement invariance across both groups. Based on Brown (2006), configural invariance, measurement invariance (e.g. metric invariance, scalar invariance) and structural invariance across the samples were tested in that order. Dimitrov (2010) has also referred to metric invariance as weak measurement invariance and scalar invariance as strong measurement invariance.

The model fit was evaluated using the chi-square statistic $\left(\chi^{2}\right)$ and other descriptive fit indices including the Comparative Fit Index (CFI; Bentler, 1990), the Root Mean Square Error of Approximation (as well as its $p$-value for $\mathrm{H}_{0}$ : rmsea $\leq .05$ ) (RMSEA; Steiger, Shapiro \& Browne, 1985) and its 90\% confidence interval), Tucker-Lewis index (TLI: Bentler-Bonett, 1980), Akaike Information Criterion (AIC: Arbuckle, 2008); Browne-Cudeck Criterion (BCC: Arbuckle, 2008) and Expected Cross-Validation Index (ECVI: Benson \& Bandalos, 1992). Hu and Bentler (1999) suggested several fit indices cutoff criteria, for a reasonably good fit between the target model and the observed data (assuming Maximum Likelihood Estimation): 1) CFI and TLI values equal to 90 or greater; 2) RMSEA values of .10 or below (See also Marôco, 2010, p. 51).

Factors' related convergent validty was assessed with the Average Variance Extracted (AVE) as proposed by Fornell \& Larcker (1981). AVE values greather than .5 were considered indicative of convergent validity (Marôco, 2010)

Criterion related validity to assess the capacity of the AAQ-II to discriminate between clinical and non clinical groups was evaluated by an independent t-test performed by SPSS Statistics. Statistiscal significance was assumed for $\mathrm{p}<.05$.

When competing models were nested, both the diference between the $\chi^{2}$ statistics and the the CFI difference were used. We used both a significant $\Delta \chi^{2}$ and CFI decrease greater than -.01 as a criterion to reject the null hypothesis of invariance (Cheung \& Rensvold, 2002). Evidence shows that $\chi^{2}$ differences are dependent on sample size (Kelloway, 1995) while CFI differences are not (Cheung \& Rensvold, 2002), besides $\chi^{2}$ differences are more susceptible to Type I error inflation, under model mispecification than CFI differences (French \& Finch, 2011).

Factors reliability were evaluated by the Cronbach's alfa measure of internal consistency as estimated by SPSS Statistics (v.18) as well as composite reliability (CR) (Fornell \& Larcker, 1981). Values of alfa and CR greater than 0.7 were indicative of acceptable reliability (Marôco \& Garcia-Marques, 2006; Marôco, 2010).

Convergent validity was estimated by $\mathrm{CR}$ values and average variance extracted (AVE); External validity of the one-factor model was estimated with a Multigroup Confirmatory Factor Analysis to analyze the invariance of this solution with independent data (Validation sample). The invariance testing included an analysis of configural invariance, measurement invariance (metric invariance and scalar invariance) and structural invariance. Several nested models were tested, with each step imposing a more restrictive level of invariance across both samples (Byrne, 2006; Brown, 2006; Dimitrov, 2010; Maroco 2010).

Finally, a Multigroup Invariance of the one-factor model was also performed to show the equivalence of the proposed model across clinical and non-clinical samples. The configural invariance model tests whether the basic factor structure is equivalent across the three samples. 
Since the satisfactory fit of the configural invariance model was shown, we proceeded to test the measurement invariance model. Measurement invariance refers to a) metric invariance- equal factor loadings across groups, b) scalar invariance- equal item intercepts across groups and, c) invariance of item uniqueness- equal item error variances/covariances across groups. After configural invariance across groups was established, testing for metric invariance was performed using $\Delta \chi^{2}$ and $\Delta C F I$ tests for two nested models. (Byrne, 2006; Brown, 2006; Dimitrov, 2010).

\section{Results}

In order to explore the factor structure underlying the items of AAQ-II (Bond et al., 2011) we conducted a Confirmatory Factorial Analysis (CFAs). Since the overall sample is large enough, it was randomly divided into two samples, to determine the replicability of the final model fitted with independent data set (the validation sample).

The 7 items of Acceptance and Action Questionnaire-II (Bond et al., 2011) were subjected to a Confirmatory Factorial Analysis (CFA). The original AAQ-II one-factor model provided an acceptable fit to the variance-covariance matrix data in the test sample (Figure 1). The model fit showed a $\chi^{2}$-value of $112.783(\mathrm{df}=14 ; p=.000)$. The overall goodness-of-fit indices showed that the proposed one-factor gave reasonable fit to the data $(\mathrm{CFI}=.950 ; \mathrm{TLI}=.925 ; \mathrm{PCFI}=.663 ; \mathrm{RMSEA}=.132, p[\mathrm{rmsea} \leq .05]<.000$; $\mathrm{AIC}=154.783 ; \mathrm{BCC}=155.627 ; \mathrm{ECVI}=.381)$.

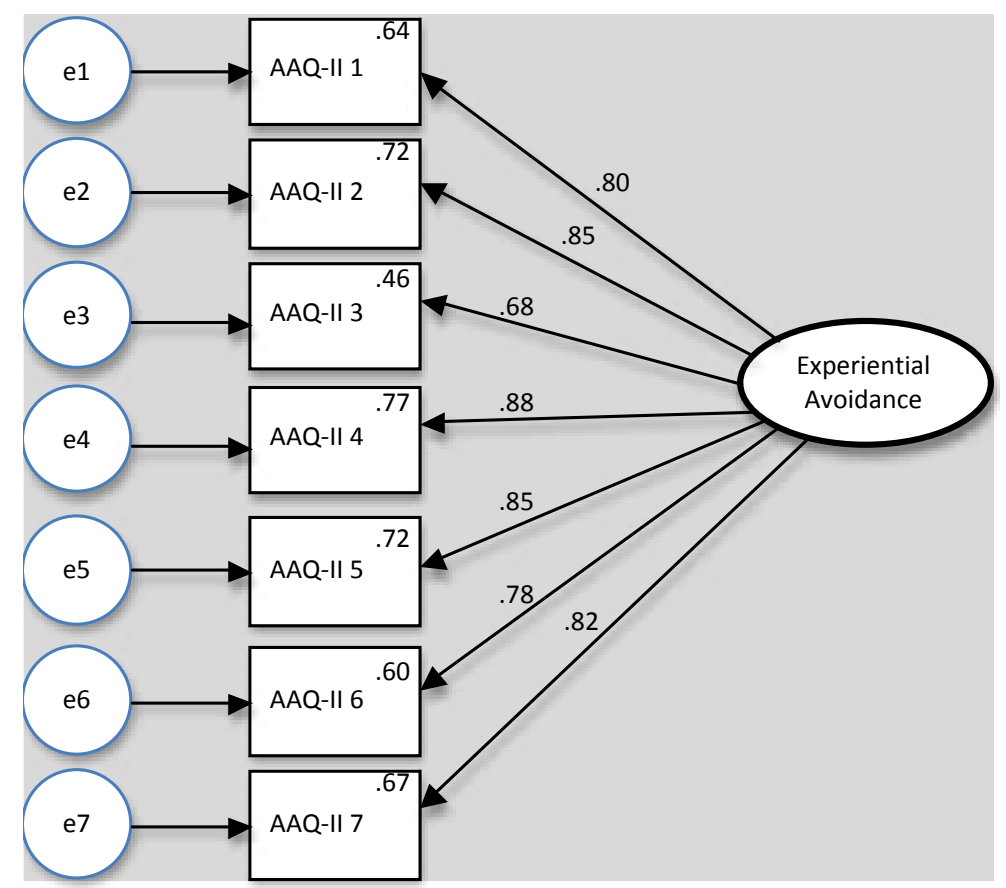

Figure 1. Standardized loadings and correlations for the one-factor model of AAQ-II. 
Our results supported a one-factor solution with 7 items loading strongly on one single factor. The obtained factor had good reliability (Cronbach's Alfa= .922)/ composite reliability of the factor proved to be of high (.95). The analysis of AVE value showed evidence of convergent validty (.74).

The criterion validity was supported by an independent t-test. An independent t-test was performed to explore the differences of the clinical and non clinical groups on experiential avoidance, as measure by the AAQ-II. There was statistically significant differences in AAQ-II score for both groups $(F(663.58)=63.905 ; p<.001)$. The results indicated that the clinical group had significant higher experiential avoidance than the general population group $(t(663.58)=10.219 ; p<.001 ; \mathrm{M}=24.72, S D=11.35 ; \mathrm{M}=17.43$, $S D=7.26$, respectively for clinical and non-clinical group).

Nested model comparisons resulted in no significant values of $\Delta \chi^{2}$ changes, which mean that there are no real differences between the models fitted to the test and validation samples (Table 2). For metric invariance model, the sequence of CFA models and respective $\Delta \chi^{2}$ tests showed a nonsignificant chi-square difference test between the configural invariance model and the metric invariance model, indicating that the factor loadings are equal across the two samples $\left(\Delta \chi^{2}(6)=6.575 ; p=.362 ; \Delta \mathrm{CFI}=.000\right)$.

A nonsignificant $\chi^{2}$ difference statistic was found between the free intercept model and the constrained intercept models model $\left(\Delta \chi^{2}(7)=4.425 ; p=.730 ; \Delta \mathrm{CFI}=-.001\right)$, suggesting that the constraints of equal item intercepts did not degraded the fit of the

Table 2. Multigroup Confirmatory Analysis used to cross validate the one-factor model proposed.

\begin{tabular}{|c|c|c|c|c|c|c|c|c|c|c|}
\hline \multicolumn{2}{|c|}{ Model } & $\chi^{2}$ & $\mathrm{df}$ & $p$ & $\operatorname{dif} \chi^{2}$ & $\mathrm{df}$ & $p$ & CFI & $\Delta \mathrm{CFI}$ & RMSEA \\
\hline \multicolumn{2}{|c|}{ Configural Invariance } & 178.502 & 28 & .000 & & & & .957 & & .088 \\
\hline \multirow{2}{*}{$\begin{array}{l}\text { Measurement } \\
\text { Invariance }\end{array}$} & Metric Invariance & 185.078 & 28 & .000 & 6.575 & 6 & .362 & .957 & .000 & .080 \\
\hline & Scalar Invariance & 189.503 & 34 & .000 & 4.425 & 7 & .730 & .958 & -.001 & .072 \\
\hline \multicolumn{2}{|c|}{ Structural Invariance } & 189.637 & 42 & .000 & .134 & 1 & .714 & .958 & .000 & .071 \\
\hline
\end{tabular}

solution and that item intercepts could be considered equal across the samples. These results support strong measurement invariance. This means that the relations between the latent factor and the external variables can be compared across the groups because a one unit change in one group would be equal to a one unit change in the other group. Given the evidence of scalar invariance, we proceeded to test the structural invariance model. A nonsignificant chi-square difference test $\left(\Delta \chi^{2}(1)=.134 ; p=.714 ; \Delta \mathrm{CFI}=.000\right)$ was found confirming the structural invariance of the AAQ-II.

The dataset results supported configural, strick measurement invariance and structural invariance of the one-factor model of AAQ-II across the test and validation samples.

Results indicate that the fit of the model was satisfactory $\left(\chi^{2}(28)=232.844 ; p=\right.$ $.000 ; \mathrm{CFI}=.930 ; \mathrm{RMSEA}=.103, p[$ rmsea $\leq .05]<.001)($ Table 3$)$.

The sequence of CFA models and respective $\Delta \chi^{2}$ tests showed a significant chisquare difference test between the configural invariance model and the metric invariance model, indicating that the factor loadings are not equal across the samples $\left(\Delta \chi^{2}(6)=\right.$ $36.391 ; p<.001)$. The CFI difference between these models was -.01 . So, it was assumed the metric invariance of the measure. This means that the constraints of equal factor loadings across the samples did not worse the model fit and the factor loadings could 
Table 3. Multigoup Invariance of the one-factor model of the Acceptance and Action Questionaire across clinical and non

\begin{tabular}{|c|c|c|c|c|c|c|c|c|c|c|}
\hline & Model & $\chi^{2}$ & $\mathrm{df}$ & $p$ & $\operatorname{dif} \chi^{2}$ & $\mathrm{df}$ & $p$ & CFI & $\Delta$ CFI & RMSEA \\
\hline \multicolumn{2}{|c|}{ Configural Invariance } & 232.844 & 28 & .000 & & 28 & & .957 & & .103 \\
\hline Measurement & Metric Invariance & 269.235 & 34 & .000 & 36.391 & 6 & .000 & .920 & -.010 & .101 \\
\hline Invariance & Scalar Invariance & 376.108 & 41 & .000 & 106.873 & 7 & .000 & .886 & -.034 & .109 \\
\hline \multicolumn{2}{|c|}{ Structural Invariance } & 413.522 & 42 & .000 & 37.414 & 1 & .000 & .874 & -.012 & .114 \\
\hline
\end{tabular}

be considered equal across the samples.

In addition to the factor loadings this analysis explored whether the item intercepts can also be considered equivalent (scalar invariance model). A significant $\chi^{2}$ difference statistic was found between the scalar invariance model and the metric invariance model $\left(\Delta \chi^{2}(7)=106.873 ; p<.001\right)$. The CFI difference between these models $(\Delta C F I=-.034)$ suggested that the constraints of equal item intercepts degraded the fit of the solution and that item intercepts could not be considered equal across the samples.

Given there was no evidence of scalar invariance, we didin't proceed to test the structural invariance across the samples which determine whether the factor variances and covariances are equal across the samples.

The multigroup analysis between clinical and normative groups, did not support the invariance of the one-factor model of the Acceptance and Action Questionnaire-II. Therefore, AAQ-II is not operating in the same way and underlying constructs have not the same factorial and metric structure among clinical and non clinical groups.

\section{Discussion}

The increasing interest in experiential avoidance combined with the faster development of research based on the theoretical approach to this process, underscores the significance of this type of investigation.

The current study sought to replicate the one-factor model identified by Bond et al. in 2011 in clinical and normative samples of the Portuguese population. Furthermore this study intended to analyze the factorial invariance of the proposed one-factor model across both clinical and general population groups, in order to examine in detail the psychometric qualities of the measure and its applicability in various contexts.

Accordingly with our first aim, we found that the original AAQ-II one-factor model provided a reasonable fit to the data. The $\chi^{2}$ test statistic was significant $\left(\chi^{2}(14)=\right.$ $112.783 ; p<.001)$. However it is known that $\chi^{2}$ test statistic is very sensitive to the sample size and may overestimate the lack of model fit (Bollen, 1989). So, several goodness-of-fit indices were also selected based upon Bollen, Hu, and Bentler (1998) and showed a good fit to data $(\mathrm{CFI}=.950 ; \mathrm{TLI}=.925 ; \mathrm{PCFI}=.663 ; \mathrm{RMSEA}=.132, p$ $[\mathrm{RMSEA} \leq .05]<.001 ; \mathrm{AIC}=154.783 ; \mathrm{BCC}=155.627 ; \mathrm{ECVI}=.381)$. It is important to notice that besides the value of RMSEA can be over estimate in unifactorial models.

Our results supported a one-factor solution with good consistency and validity. Convergent validity was supported by a higher composite reliability value and also by the high average variance extratcted. Criterion validity was supported by the results of an independent t-test that showed that AAQ-II scores were significantly different etween clinical and non clinical groups. 
Concerning concorrent validity, Bond et al. (2011) showed higher scores of AAQII related to similar constructs such as thought suppression. In Bond et al. (2011) the divergent validity was shown by no associations of the AAQ-II scores and theoretically distinct constructs.

The current study also supported external validity by a multigroup confirmatory analysis, showing configural, strick measurement and structural invariance of the AAQ-II one-factor solution with independent data.

It is important to notice that as far as we know this is the first study performed to clearly understand the AAQ-II factorial structure in a large Portuguese sample with both clinical and normative population groups. This results add to Bond et al. (2011) who found a satisfactory structure, with good reliablility and validity.

Testing for model fit relates to the structural aspect of variability but not tap into the generalizability aspect of the validity (Dimitrov, 2010). So, the second aim of the current study was to examine the multigroup invariance of the one-factor model proposed for AAQ-II across both clinical and normative population groups. Our data only support configural invariance.

Specifically, a baseline model was established (this means the most meaningfull and best fitting to data for both groups). Based on our results, it can be assume that the one-factor model proposed has equivalency and the constructs have similar meanings across the two groups under study. Besides the one-factor model have both similar meanings and the same structure, the measurement model in clinical and nonclinical groups was not the same. This is to say that at the level of measurement invariance the relationships between the latent factor and external variables can not be compared across both groups.

Thus our findings did not support measurement invariance and so the generalizability aspect of the validity across clinical and nonclinical groups cannot be supported, our findings add to the existent knowledge in this area by confirming the one-factor model of AAQ-II as the best structure, with good psychometric properties.

Since previous work has identified the toxic influences of experiencial avoidance as a core mechanism in the development and maintenance of several clinical disorders, it is possible that AAQ-II does not operate equivalently across clinical and nonclinical groups. In fact, our findings showed that experiential avoidance has the same meaning for both groups but the factor loadings across them are not equal. A closely analysis of the factor loadings clearly show significantly higher factor loadings across clinical group in comparision with nonclinical group. Our results show the usefulness of this new version of AAQ-II in both clinical and non clinical samples. We assume that besides in both clinical and nonclinical samples experiential avoidance is strongly correlated with measures of general pathology, these findings reinforeced that experiential avoidance is much more prevalent in rigid and inflexible contexts where time, effort and energy is spent to managing, controlling or struggling with unwanted private events.

When interpreting our results, one should keep in mind that the present study is subject to several limitations. It most be notice that the use of a non probabilistic sample (by convenience) limits the generalization of our conclusions.

Besides the present findings evidencing that AAQ-II has good psychometric properties 
and its stable across independent samples, future research is need to fully examine the multigroup invariance of the one-factor model across clinical and nonclinical/ normative groups. In fact, AAQ-II was not designed as a tool for diagnosing mental disorders but to assess a specific model of psychopatology that emphasizes psychological inflexibility.

We replicate the factor structure of one-factor model of experiential avoidance developed by Bond et al. (2011). Our findings support a one-factor structure of AAQ-II with strong evidence of psychometric validity. This study adds to previous knowledge by showing that the proposed one-factor model is a measure that not only shows adequate psychometric properties but also measures the same psychological construct in pathological and normative groups. Findings showed that experiential avoidance has the same meaning for both groups but the factor loadings across them were not equal, with significantly higher factor loadings across clinical group in comparision with nonclinical group. Future research should investigate the factorial invariance within several groups of clinical disorders.

Regarding the demographic variables, there were significant sex differences regarding to marital status, socio-economic status and age. From the authors' point of view the differences relating to marital and socio-economic status could be justified by the heterogeneity of samples under study (rheumatoid arthritis, infertility and general population). However, it would be important, in future studies, to analyze theses age differences in an attempt to meet and understand the differences in experiential avoidance levels based on clinical membership.

According to Flaxman, Blackledge, and Bond (2011), the human capacity for experiential avoidance is significant for two mais reasons. First, many experientially avoidant behaviours either cause physical harm and compound the problem that engendered them in the first place. In fact, there are many instances in that experiential avoidance may offer some initial relief, but make our problems and our distress worse over the long run. Second, many instances of Experiential avoidance prevent one from living in a meaningful, purposeful and vital way. When life is lived in such a manner, life satisfaction and well-being would be expected to decrease over the long run.

\section{REFERENCES}

Arbuckle J (2008). AMOS 17 Users' Guide. Chicago, IL: SPSS.

Benson J \& Bandalos D (1992). Second-Order Confirmatory Factor Analysis of the Reactions to Tests Scale with Cross-Validation. Multivariate Behavioral Research, 27, 459-487. doi:10.12.07/ s15327906mbr2703_8

Bentler P (1990). Comparative fit indexes in structural models. Psychological Bulletin, 107, 238-246. doi:10.1037/0033-2909.107.2.238

Bentler P \& Bonett D (1980). Significance tests and goodness of fit in the analysis of covariance structures. Psychological Bulletin, 88, 588-606. doi:10.1037/0033-2909.88.3.588

Bollen K (1989). Structural equations with latent variables. New York: Wiley. 
Bond F, Hayes S, Baer R, Carpenter K, Guenole N, Orcutt H, Waltz T \& Zettle R (2011). Preliminary psychometric properties of the Acceptance and Action Questionnaire-II: A revised measure of psychological inflexibility and experiential avoidance. Behavior Therapy, 42, 676-688. doi:10.1016/j.beth.2011.03.007

Brown T (2006). Confirmatory Factor Analysis for Applied Research. New York: The Guilford Press.

Byrne B (2006). Structural equation modeling with EQS: Basic concepts, aplications, and programming. Mahwah, NH: Erlbaum.

Cheung G \& Rensvold R (2002). Evaluating goodness-of-fit indexes for testing measurement invariance. Structural Equation Modeling, 9, 233-255. doi:10.1207/S15328007SEM0902_5

Dimitrov D (2010). Testing for Factorial Invariance in the Context of Construct Validation. Measurement and Evaluation in Counseling and Development, 43, 121-149. doi: 10.1177/0748175610373459

Flexman P, Blackledge J \& Bond F (2011). Acceptance and Commitment Therapy. New York: Routledge.

French B \& Finch W (2011). Model misspecification and invariance testing using confirmatory factor analytic procedures. Journal of Experimental Education, 79, 404-428. doi:10.1080/00220973 .2010 .517811

Fornell C \& Larcker D (1981). Evaluation SEM with unobserced variables and measurement error. Journal of Marketing Research, 18, 39-50.doi:10.2307/3151312

Forsyth J, Eifert G \& Barrios V (2006). Fear conditioning in an emotion regulation context: A fresh perspective on the origins of anxiety disorders. In M G Craske, D Hermans \& D Vansteenwegen (Eds), Fear and learning: From basic processes to clinical applications (pp. 133-153). Washington, DC: American Psychological Association.

Hayes S (2004). Acceptance and Commitment Therapy, Relational Frame Theory, and the Third Wave of Behavioral and Cognitive Therapies. Behavior Therapy, 35, 639-665. doi:10.1016/s00057894(04)80013-3

Hayes S \& Duckworth M (2006). Acceptance and commitment therapy and traditional cognitive behavior therapy approaches to pain. Cognitive and Behavioral Practice, 17, 185- 187.

Hayes S, Strosahl K \& Wilson K (1999). Acceptance and Commitment Therapy. An experiential approach to behaviour change. New York: The Guilford Press.

Hayes S, Strosahl K, Wilson K, Bissett R, Pistorello J, Toarmino D et al (2004). Measuring experiencial avoidance: A preliminary test of a working model. The Psychological Record, 54, 5543-578.

Hayes S, Wilson K, Gifford E, Follette V \& Strosahl (1996). Experiential avoidance and behavioral disorders: A functional dimensional approach to diagnosis and treatment. Journal of Consulting and Clinical Psychology, 64(6), 1152-1168. doi:10.1037/0022-006x.61152

Hu L \& Bentler P (1999). Cutoff criteria for fit indexes in covariance structure analysis: Conventional criteria versus new alternatives. Structural Equation Modeling, 6, 1-55. doi:10.1080/10705519909540118

Kashdan T, Barrios V, Forsyth J \& Steger M (2006). Experiential avoidance as a generalized psychological vulnerability: comparisons with coping and emotion regulation strategies. Behaviour Research and Therapy, 44, 1301-1320. doi:10.1016/j.brat.2005.10.003

Kelloway K (1995). Structural equation modelling in perspective. Journal of Organizational Behavior, 16, 215-224. doi:10.1002/job.4030160304

Marôco J (2010). Análise de Equações Estruturais. Fundamentos teóricos, Software e Aplicações [Analysis of Structural Equations. Theoretical foundations, Sofware and Applications]. Pêro Pinheiro: ReportNumber.

Marôco J \& Garcia-Marques T (2006). Qual a fiabilidade do alfa de Cronbach? Questões antigas e soluções modernas? [How reliable Cronbach's alpha? Old issues and modern solutions?] Laboratório de Psicologia, 4, 65-90 .

Pinto-Gouveia J, Gregório S, Dinis A \& Xanier A (2012). Experiential Avoidance in Clinical and NonClinical Samples: AAQ-II Portuguese Version. International Journal of Psychology \& Psycho- 
logical Therapy, 12, 139-156.

Rüsch N, Scjhield S, Corrigan P, Leihener F, Jacob G, Olschewski M, Lieb K, et al (2008). Predictors of dropout from inpatient dialectical behavioral therapy among women with borderline personality disorder. Journal of Behavior Therapy and Experiential Psychiatry, 35, 497-503.

Sloan D (2004). Emotion regulation in action: emotional reactivity in experiential avoidance. Behaviour Research and Therapy, 42, 1257-1270. doi:10.1016/j.rat.2003.08.006

Steiger J, Shapiro A \& Browne M (1985). On the multivariaye asymptotic distribution of sequential chi-square statistics. Psychometrika, 50, 253-263. doi:10.1007/BF02294104

Received, December 13, 2013

Final Acceptance, June 20, 2014 